\title{
Meromorphic solutions of Painlevé III difference equations with Borel exceptional values
}

\section{Jilong Zhang*}

\section{"Correspondence:}

jilongzhang2007@gmail.com

LMIB and School of Mathematics \&

Systems Science, Beihang

University, Beijing, 100191, P.R. China

\begin{abstract}
In this paper, we investigate the properties of meromorphic solutions of Painlevé III difference equations. In particular, the difference equation $\bar{w} \underline{w} w(w-1)=\mu$ with $\mu$ being a non-zero constant is studied. We show that the rational solutions of the equation assume only one form and the transcendental solutions have at most one Borel exceptional value. We also show that the difference equation

$\bar{w} \underline{w}(w-1)^{2}=(w-\lambda)^{2}$ does not have nonconstant rational solution, where $\lambda(\neq 0,1)$ is a constant.
\end{abstract}

MSC: $30 \mathrm{D} 35 ; 39 \mathrm{~A} 10$

Keywords: meromorphic solution; difference; finite order

\section{Introduction}

Let $w$ be a meromorphic function in the complex plane. The $z$-dependence is supposed by writing $\bar{w} \equiv w(z+1)$ and $\underline{w} \equiv w(z-1)$. We assume that the reader is familiar with the standard notations and results of Nevanlinna value distribution theory (see, e.g., [1-3]). $\rho(w)$, $\lambda(w)$ and $\lambda(1 / w)$ denote the order, the exponents of convergence of zeros and poles of $w$, respectively. Furthermore, we denote by $S(r, w)$ any quantity satisfying $S(r, w)=o(T(r, w))$ for all $r$ outside of a set with finite logarithmic measure and by

$$
\mathcal{S}(w)=\{\alpha \text { meromorphic }: T(r, \alpha)=S(r, w)\}
$$

the field of small functions with respect to $w$. A meromorphic solution $w$ of a difference equation is called admissible if all coefficients of the equation are in $\mathcal{S}(w)$. For example, if a difference equation has only rational coefficients, then all non-rational meromorphic solutions are admissible; if an admissible solution is rational, then all the coefficients must be constants.

Recently, with the development of Nevanlinna value distribution theory on difference expressions [4-6], Halburd and Korhonen [7] gave the full classification of the family including Painlevé I and II difference equations. As for the family including Painlevé III difference equations, we recall the following.

C2014 Zhang; licensee Springer. This is an Open Access article distributed under the terms of the Creative Commons Attribution License (http://creativecommons.org/licenses/by/2.0), which permits unrestricted use, distribution, and reproduction in any medium, provided the original work is properly cited. 
Theorem A ([8]) Assume that the equation

$$
\bar{w} \underline{w}=R(z, w)
$$

has an admissible meromorphic solution $w$ of hyper-order less than one, where $R(z, w)$ is rational and irreducible in $w$ and meromorphic in $z$, then either $w$ satisfies a difference Riccati equation

$$
\bar{w}=\frac{\alpha w+\beta}{w+\gamma}
$$

where $\alpha, \beta, \gamma \in \mathcal{S}(w)$ are algebroid functions, or equation (1.1) can be transformed to one of the following equations:

$$
\begin{aligned}
& \bar{w} \underline{w}=\frac{\eta w^{2}-\lambda w+\mu}{(w-1)(w-v)}, \\
& \bar{w} \underline{w}=\frac{\eta w^{2}-\lambda w}{(w-1)}, \\
& \bar{w} \underline{w}=\frac{\eta(w-\lambda)}{(w-1)}, \\
& \bar{w} \underline{w}=h w^{m} .
\end{aligned}
$$

In (1.2a), the coefficients satisfy $\kappa^{2} \bar{\mu} \mu=\mu^{2}, \bar{\lambda} \mu=\kappa \underline{\lambda} \bar{\mu}, \kappa \overline{\bar{\lambda}} \underline{\underline{\lambda}}=\underline{\kappa} \lambda \bar{\lambda}$ and one of the following:
(1) $\eta \equiv$
$\bar{v} \underline{v}=1$,
$\kappa=v$
(2) $\bar{\eta}=\eta=v, \quad \kappa \equiv 1$.

In (1.2b), $\eta \bar{\eta}=1$ and $\overline{\bar{\lambda}} \underline{\lambda}=\lambda \bar{\lambda}$. In (1.2c), the coefficients satisfy one of the following:

(1) $\eta \equiv 1$ and either $\lambda=\bar{\lambda} \underline{\lambda}$ or $\bar{\lambda}^{[3]} \underline{\lambda}_{[3]}=\overline{\bar{\lambda}} \underline{\underline{\lambda}}$;

(2) $\bar{\lambda} \underline{\lambda}=\overline{\bar{\lambda}} \underline{\underline{\lambda}}, \bar{\eta} \bar{\lambda}=\overline{\bar{\lambda}} \underline{\underline{\eta}}, \eta \underline{\eta}=\overline{\bar{\eta}} \underline{\underline{\eta}}[3]$;

(3) $\overline{\bar{\eta}} \underline{\eta}=\eta \underline{\eta}, \lambda=\underline{\eta}$;

(4) $\overline{\bar{\lambda}}^{\overline{\overline{[3}}]} \underline{\lambda}_{[3]}=\overline{\bar{\lambda}} \underline{\underline{\lambda}} \lambda, \eta \lambda=\overline{\bar{\eta}} \underline{\underline{\eta}}$,

where $\bar{\lambda}^{[3]} \equiv \lambda(z+3)$ and $\underline{\lambda}_{[3]} \equiv \lambda(z-3)$. In (1.2d), $h \in \mathcal{S}(w)$ and $m \in \mathbb{Z},|m| \leq 2$.

In 2010, Chen and Shon [9] started the topic of researching the properties of finite-order meromorphic solutions of difference Painlevé I and II equations. In fact, they showed that if $w$ is a transcendental finite-order meromorphic solution of the equation

$$
\bar{w}+\underline{w}=\frac{\left(a_{1} z+a_{2}\right) w+a_{3}}{1-w^{2}} \quad \text { or } \quad \bar{w}+\underline{w}=\frac{a_{4} z+a_{5}}{w}+a_{6},
$$

where $a_{j}(1 \leq j \leq 6)$ are constants with $a_{1} a_{3} a_{4} \neq 0$, then $w$ has at most one non-zero finite Borel exceptional value.

The present author and Yang [10] improved the above result and verified that $w$ does not have any Borel exceptional value. And they also considered the difference Painlevé III equations (1.2d) with the constant coefficients. The difference equations $(1.2 \mathrm{~b})$ and $(1.2 \mathrm{c})$ are studied by the present author in the following. 
Theorem B ([11]) If $w$ is a transcendental finite-order meromorphic solution of

$$
\bar{w} \underline{w}(w-1)=\eta w \quad \text { or } \quad \bar{w} \underline{w}(w-1)=w^{2}-\lambda w,
$$

where $\eta(\neq 0), \lambda(\neq 0,1)$ are constants, then

(i) $\lambda(w)=\rho(w)$;

(ii) $w$ has at most one non-zero Borel exceptional value for $\rho(w)>0$.

The purpose of this paper is to study the remaining difference equation (1.2a) with constant coefficients. As it is complicated to discuss meromorphic solutions of (1.2a) when $\lambda \mu \neq 0$, we will consider the following special cases.

Theorem 1.1 Suppose that $w$ is a nonconstant rational solution of

$$
\bar{w} \underline{w} w(w-1)=\mu,
$$

where $\mu$ is a non-zero constant. Then $\mu=-\frac{3^{3}}{4^{4}}$ and $w$ assumes the only form of

$$
w(z)=\frac{3(z+b)^{2}}{4(z+b+1)(z+b-1)}
$$

with any constant $b$.

Remark Equation (1.3) is a special case of (1.2a) in the option (2) as $\eta=\lambda=v=0$. From the proof of Theorem 1.1, we shall see that the degrees of numerator and denominator of $w(z)$ must be 2 . Here, the coefficient $\frac{3}{4}$ is determined. We think that it is because there is a -1 in (1.3).

Theorem 1.2 Suppose that $w$ is a transcendental finite-order meromorphic solution of (1.3). Then

(i) $\lambda(w)=\rho(w)$;

(ii) $w$ has at most one Borel exceptional value for $\rho(w)>0$.

Example 1.3 The function $w_{1}(z)=\frac{3\left(e^{2 \pi i z}+z\right)^{2}}{4\left(e^{2 \pi i z}+z+1\right)\left(e^{2 \pi i z}+z-1\right)}$ is a solution of the difference equation $\bar{w} \underline{w} w(w-1)=-\frac{3^{3}}{4^{4}}$. It is easy to see that $\lambda\left(w_{1}\right)=\rho\left(w_{1}\right)$. Since $w_{1}-\frac{3}{4}=\frac{3}{4\left(e^{2 \pi i z}+z+1\right)\left(e^{2 \pi i z}+z-1\right)}$, then $\frac{3}{4}$ is a Picard exceptional value of $w_{1}$. This shows that the conclusions of Theorem 1.2 may occur.

Remark In $w_{1}$ in Example 1.3, the function $e^{2 \pi i z}$ can be replaced by any finite-order function with period 1 . For example, $\sin (2 \pi z), \tan (\pi z)$ and so on.

Theorem 1.4 Suppose that $w$ is a nonconstant meromorphic solution of

$$
\bar{w} \underline{w}(w-1)^{2}=(w-\lambda)^{2},
$$

where $\lambda(\neq 0,1)$ is a constant. Then $w$ must be transcendental. 
Theorem 1.5 Suppose that $w$ is a transcendental finite-order meromorphic solution of (1.4). If $a$ and $b$ are two Borel exceptional values of $w$, then

(i) $a+b=2, a b=\lambda$;

(ii) $\bar{w}=\underline{w}$;

(iii) $w$ satisfies the difference Riccati equation $\bar{w}=\frac{w-\lambda}{w-1}$.

Example 1.6 The transcendental function $w_{2}(z)=\frac{3 e^{i \pi z}-1}{e^{i \pi z}+1}$ is a solution of both the difference equation $\bar{w} \underline{w}(w-1)^{2}=(w+3)^{2}$ and the Riccati equation $\bar{w}=\frac{w+3}{w-1}$. Noting that $w_{2}(z)$ has two Picard exceptional values 3 and -1 , we see that the conclusions in Theorem 1.5 may occur.

\section{Some lemmas}

Halburd and Korhonen [5] and Chiang and Feng [4] investigated the value distribution theory of difference expressions, a key result of which is a difference analogue of the logarithmic derivative lemma. With the help of the lemma, the difference analogues of the Clunie and Mohon'ko lemmas are obtained.

Lemma 2.1 ([6]) Let $f$ be a transcendental meromorphic solution of finite order $\rho$ of a difference equation of the form

$$
U(z, f) P(z, f)=Q(z, f)
$$

where $U(z, f), P(z, f)$ and $Q(z, f)$ are difference polynomials such that the total degree $\operatorname{deg}_{f} U(z, f)=n \operatorname{in} f(z)$ and its shifts, and $\operatorname{deg}_{f} Q(z, f) \leq n$. If $U(z, f)$ contains just one term of maximal total degree in $f(z)$ and its shifts, then, for each $\varepsilon>0$,

$$
m(r, P(z, f))=O\left(r^{\rho-1+\varepsilon}\right)+S(r, f),
$$

possibly outside of an exceptional set of finite logarithmic measure.

Lemma $2.2([5,6])$ Let $w$ be a transcendental meromorphic solution of finite order of the difference equation

$$
P(z, w)=0,
$$

where $P(z, w)$ is a difference polynomial in $w(z)$. If $P(z, a) \not \equiv 0$ for a meromorphic function $a \in \mathcal{S}(w)$, then

$$
m\left(r, \frac{1}{w-a}\right)=S(r, w) .
$$

We conclude this section by the following lemma.

Lemma 2.3 (see, e.g., [3, pp.79-80]) Let $f_{j}(j=1, \ldots, n)(n \geq 2)$ be meromorphic functions, $g_{j}(j=1, \ldots, n)$ be entire functions. If

(i) $\sum_{j=1}^{n} f_{j}(z) e^{g_{j}(z)} \equiv 0$;

(ii) $g_{h}(z)-g_{k}(z)$ is not a constant for $1 \leq h<k \leq n$;

(iii) $T\left(r, f_{j}\right)=S\left(r, e^{g_{h}(z)-g_{k}(z)}\right)$ for $1 \leq j \leq n$ and $1 \leq h<k \leq n$, then $f_{j}(z) \equiv 0(j=1, \ldots, n)$. 


\section{Proofs of theorems}

Proof of Theorem 1.1 Suppose that $w=\frac{P(z)}{Q(z)}$, where $P(z)$ and $Q(z)$ are relatively prime polynomials with degrees $p$ and $q$ respectively. It follows from (1.3) that

$$
\frac{P(z+1)}{Q(z+1)} \frac{P(z-1)}{Q(z-1)} \frac{P(z)}{Q(z)} \frac{P(z)-Q(z)}{Q(z)}=\mu .
$$

Without loss of generality, we assume that the coefficients of the highest degree terms of $P(z)$ and $Q(z)$ are $a$ and 1 respectively. Let $s=p-q$.

If $s>0$, then $\frac{P(z)}{Q(z)}=a z^{s}(1+o(1))$ as $z$ tends to infinity, where $a$ is a non-zero constant. And (3.1) gives

$$
a^{3}(z+1)^{s}(z-1)^{s} z^{s}(1+o(1))\left(a z^{s}(1+o(1))-1\right)=\mu,
$$

which is a contradiction since the left-hand side of the above equation goes to infinity as $r$ tends to infinity, while the right-hand side is a constant.

If $s<0$, we have $\frac{P(z)}{Q(z)}=o(1)$ and $\frac{P(z \pm 1)}{Q(z \pm 1)}=o(1)$ as $z$ tends to infinity. Equation (3.1) yields

$$
o(1)=\mu, \quad z \rightarrow \infty
$$

which contradicts $\mu \neq 0$.

Thus $s=0$ and $p=q$. Noting that the zeros of $Q(z)$ are not the zeros of $P(z)$ and $P(z)-$ $Q(z)$, we get from (3.1) that all the zeros of $Q^{2}(z)$ are the zeros of $P(z+1) P(z-1)$. As the degrees of $Q^{2}(z)$ and $P(z+1) P(z-1)$ are both $2 p$, we obtain

$$
\begin{aligned}
& P(z+1) P(z-1)=a^{2} Q^{2}(z), \\
& P(z)(P(z)-Q(z))=a(a-1) Q(z+1) Q(z-1) .
\end{aligned}
$$

Now, we aim to prove that the orders of all the zeros of $P(z)$ are even. Otherwise, assume that $z_{0}$ is a zero of $P(z)$ with the order $k$, and $k$ is an odd integer. Then $P(z)$ has the term $\left(z-z_{0}\right)^{k}$, and $P(z+1) P(z-1)$ has the term

$$
\left(z-z_{0}+1\right)^{k}\left(z-z_{0}-1\right)^{k}
$$

caused by $z_{0}$. It means that $z_{0}-1$ and $z_{0}+1$ are both zeros of $P(z+1) P(z-1)$ with the order at least $k$.

On the other hand, it follows from (3.3) that $Q(z+1) Q(z-1)$ has the term $\left(z-z_{0}\right)^{k}$ exactly. Suppose that $Q(z+1)$ and $Q(z-1)$ have the terms $\left(z-z_{0}\right)^{m}$ and $\left(z-z_{0}\right)^{l}$ respectively, where $m$ and $l$ are non-negative integers satisfying $m+l=k$. Then $Q(z)$ has term $\left(z-z_{0}-1\right)^{m}(z-$ $\left.z_{0}+1\right)^{l}$ exactly, i.e., $Q^{2}(z)$ has the term $\left(z-z_{0}-1\right)^{2 m}\left(z-z_{0}+1\right)^{2 l}$. By (3.2), $P(z+1) P(z-1)$ has the term

$$
\left(z-z_{0}+1\right)^{2 l}\left(z-z_{0}-1\right)^{2 m}
$$

Without loss of generality, assume that $m<l$. Obviously, $2 m<k$ and $2 l>k$. Then $z_{0}+1$ is a zero of $P(z+1) P(z-1)$ with the order $2 m<k$, which is a contradiction by (3.4). Therefore, all the zeros of $P(z)$ have even orders. 
Denote $P(z)=a r^{2}(z)$, where

$$
r(z)=z^{n}+A_{n-1} z^{n-1}+A_{n-2} z^{n-2}+\cdots+A_{1} z+A_{0} .
$$

Noting that the coefficient of the highest degree term of $Q(z)$ is 1 , it is easy to see from (3.2) and (3.3) that $Q=\bar{r} \underline{r}$ and $a r^{2}-\bar{r} \underline{r}=(a-1) \underline{\bar{r}} \underline{\underline{r}}$. Let

$$
\phi=a r^{2}-\bar{r} \underline{r}-(a-1) \overline{\bar{r}} \underline{\underline{r}} .
$$

Then $\phi(z) \equiv 0$. If $\operatorname{deg} r=n \geq 2$, substituting $r$ by (3.6) in the right-hand side of the last equation, we have that the coefficients of terms $z^{2 n-2}, z^{2 n-3}$ and $z^{2 n-4}$ of $\phi(z)$ are

$$
\begin{aligned}
& B_{2 n-2}=n(4 a-3), \\
& B_{2 n-3}=2(n-1)(4 a-3) A_{n-1}, \\
& B_{2 n-4}=(15-16 a) C_{n}^{2}+2(n-3)(4 a-3) A_{n-2}+(n-1)(4 a-3) A_{n-1}^{2} .
\end{aligned}
$$

We deduce from $B_{2 n-2}=0$ that $a=\frac{3}{4}$. And then $B_{2 n-4}=3 C_{n}^{2} \neq 0$, thus deg $r=1$. Let $r=z+b$. Then

$$
w(z)=\frac{P(z)}{Q(z)}=\frac{3(z+b)^{2}}{4(z+b+1)(z+b-1)},
$$

and $\mu=-\frac{3^{3}}{4^{4}}$.

Proof of Theorem 1.4 Assume to the contrary that $w$ is a nonconstant rational function. Denote $w=\frac{P(z)}{Q(z)}$, where $P(z)$ and $Q(z)$ are relatively prime polynomials with degrees $p$ and $q$ respectively. It follows from (1.4) that

$$
\frac{P(z+1)}{Q(z+1)} \frac{P(z-1)}{Q(z-1)}\left(\frac{P(z)}{Q(z)}-1\right)^{2}=\left(\frac{P(z)}{Q(z)}-\lambda\right)^{2} .
$$

By the same reasoning as in the proof of Theorem 1.1, we have $p=q$. We also assume that the coefficients of the highest degree terms of $P(z)$ and $Q(z)$ are $a$ and 1 respectively. Let $z \rightarrow \infty$, it follows from (3.7) that

$$
a^{2}(a-1)^{2}=(a-\lambda)^{2}
$$

Obviously, $a \notin\{0,1, \lambda\}$. Rewriting (3.7) as

$$
\frac{P(z+1)}{Q(z+1)} \frac{P(z-1)}{Q(z-1)}=\left(\frac{P(z)-\lambda Q(z)}{P(z)-Q(z)}\right)^{2},
$$

and noting that the degrees of $P(z)-\lambda Q(z)$ and $P(z)-Q(z)$ are both $p$, we have

$$
\begin{aligned}
& (a-\lambda)^{2} P(z+1) P(z-1)=a^{2}(P(z)-\lambda Q(z))^{2}, \\
& (a-1)^{2} Q(z+1) Q(z-1)=(P(z)-Q(z))^{2} .
\end{aligned}
$$


Suppose that $z_{0}$ is a zero of $P(z)$ with the order $k$, and $k$ is an odd integer. Then $z_{0}-1$ is a zero of $P(z+1)$ with the order $k$. However, since all the zeros of $P(z+1) P(z-1)$ have even orders by (3.8), $z_{0}-1$ must be a zero of $P(z-1)$ with the odd order $l$. Thus, $z_{0}-2$ is a zero of $P(z)$ with the odd order $l$. Therefore, $z_{0}-2 m$ are all zeros of $P(z)$ by induction, which is impossible. Then all the zeros of $P(z)$ have even orders. Similarly, all the zeros of $Q(z)$ have even orders too.

Denote $P(z)=a r^{2}(z)$ and $Q(z)=t^{2}(z)$, where

$$
\begin{aligned}
& r(z)=z^{n}+A_{n-1} z^{n-1}+A_{n-2} z^{n-2}+\cdots+A_{1} z+A_{0}, \\
& t(z)=z^{n}+B_{n-1} z^{n-1}+B_{n-2} z^{n-2}+\cdots+B_{1} z+B_{0} .
\end{aligned}
$$

We obtain from (3.9) and (3.10) that

$$
\begin{aligned}
& (a-\lambda) \bar{r} \underline{r}=a r^{2}-\lambda s^{2}, \\
& (a-1) \bar{s} \underline{s}=a r^{2}-s^{2} .
\end{aligned}
$$

Substituting $r$ and $t$ by (3.11) and (3.12) respectively in the last two equations and comparing the coefficients of terms $z^{2 n-1}$ and $z^{2 n-2}$, we have $A_{n-1}=B_{n-1}$ and

$$
\begin{aligned}
& -n(a-\lambda)=2 \lambda\left(A_{n-2}-B_{n-2}\right), \\
& -n(a-1)=2 a\left(A_{n-2}-B_{n-2}\right) .
\end{aligned}
$$

It is easy to see that $A_{n-2} \neq B_{n-2}$ by $a \neq 1$. Then

$$
a(a-\lambda)=\lambda(a-1)
$$

Combining the above equation with (3.8), we get that $a=0$ or $a=1$, a contradiction.

Proof of Theorem 1.5 Rewriting the difference equation (1.4) as

$$
\bar{w} \underline{w} w^{2}=2 \bar{w} \underline{w} w-\bar{w} \underline{w}+(w-\lambda)^{2},
$$

we have from Lemma 2.1 that $m(r, w)=S(r, w)$, then $N(r, w)=T(r, w)+S(r, w)$ and $\infty$ is not the Borel exceptional value of $w$. Thus, $a$ and $b$ are finite complex numbers.

Let

$$
P(z, w)=\bar{w} \underline{w}(w-1)^{2}-(w-\lambda)^{2} .
$$

It is easy to see that $P(z, 0)=-\lambda^{2} \neq 0$. Lemma 2.2 tells us that

$$
N\left(r, \frac{1}{w}\right)=T(r, w)+S(r, w)
$$

and then $a b \neq 0$. 
Set

$$
f(z)=\frac{w(z)-a}{w(z)-b} .
$$

Then $\rho(f)=\rho(w), \lambda(f)=\lambda(w-a)<\rho(f)$ and $\lambda(1 / f)=\lambda(w-b)<\rho(f)$. Since $f$ is of finite order, we suppose that

$$
f(z)=g(z) e^{d z^{n}}
$$

where $d(\neq 0)$ is a constant, $n(\geq 1)$ is an integer, $g(z)$ is meromorphic and satisfies

$$
\rho(g)<\rho(f)=n
$$

Then

$$
f(z+1)=g(z+1) g_{1}(z) e^{d z^{n}}, \quad f(z-1)=g(z-1) g_{2}(z) e^{d z^{n}},
$$

where $g_{1}(z)=e^{n d z^{n-1}+\cdots+d}$ and $g_{2}(z)=e^{-n d z^{n-1}+\cdots+(-1)^{n} d}$.

We get from (3.13) that $w=\frac{b f-a}{f-1}$. By (1.4) and (3.16), we have

$$
A(z) e^{4 d z^{n}}+B(z) e^{3 d z^{n}}+C(z) e^{2 d z^{n}}+D e^{d z^{n}}+E=0,
$$

where

$$
\begin{aligned}
& A(z)= {\left[b^{2}(b-1)^{2}-(b-\lambda)^{2}\right] g^{2} \bar{g} g_{1} \underline{g} g_{2}, } \\
& B(z)= {\left[2 b^{2}(b-1)(1-a)-2(b-\lambda)(\lambda-a)\right] g \bar{g} g_{1} g g_{2} } \\
&+\left[(b-\lambda)^{2}-a b(b-1)^{2}\right] g^{2}\left(\bar{g} g_{1}+\underline{g} g_{2}\right), \\
& C(z)= {\left[a^{2}(b-1)^{2}-(b-\lambda)^{2}\right] g^{2}+\left[b^{2}(a-1)^{2}-(a-\lambda)^{2}\right]\left(\bar{g} g_{1} g g_{2}\right) } \\
&+2[(b-\lambda)(\lambda-a)+a b(b-1)(a-1)] g\left(\bar{g} g_{1}+\underline{g g} g_{2}\right), \\
& D(z)=2\left[a^{2}(b-1)(1-a)-(b-\lambda)(\lambda-a)\right] g+\left[(\lambda-a)^{2}-a b(1-a)^{2}\right]\left(\bar{g} g_{1}+g g_{2}\right), \\
& E=a^{2}(a-1)^{2}-(a-\lambda)^{2} .
\end{aligned}
$$

From (3.15), we apply Lemma 2.3 to (3.17), resulting in all the coefficients vanish. We deduce from $A(z)=0$ and $E=0$ that

$$
a^{2}(a-1)^{2}=(a-\lambda)^{2}, \quad b^{2}(b-1)^{2}=(b-\lambda)^{2} .
$$

Thus

$$
a-\lambda= \pm a(a-1), \quad b-\lambda= \pm b(b-1) .
$$

Define $G=g, G_{1}=\bar{g} g_{1}$ and $G_{2}=g g_{2}$. We discuss the following three cases. 
Case 1. Suppose that

$$
a-\lambda=a(a-1), \quad b-\lambda=b(b-1) .
$$

It follows from the above equations that $a$ and $b$ are distinct zeros of the equation

$$
z^{2}-2 z+\lambda=0
$$

Then

$$
a+b=2, \quad a b=\lambda .
$$

From $B(z)=0, D(z)=0$ and (3.18), we have

$$
\begin{aligned}
& 2 b(b-1)(1-a) G_{1} G_{2}=b(b-1)^{2} G\left(G_{1}+G_{2}\right), \\
& 2 a(b-1)^{2}(a-b) G=-a(b-1)^{2}(a-b)\left(G_{1}+G_{2}\right) .
\end{aligned}
$$

If $b=1$, we get from (3.18) that $a=1$, which is a contradiction to $a \neq b$. Then $b \neq 1$. Noting that $a b \neq 0$, the last two equations yield

$$
\begin{aligned}
& -2 G_{1} G_{2}=G\left(G_{1}+G_{2}\right), \\
& -2 G=G_{1}+G_{2} .
\end{aligned}
$$

Combining with the last two equations, we have $4 G_{1} G_{2}=\left(G_{1}+G_{2}\right)^{2}$, which means, with $-2 G=G_{1}+G_{2}$, that

$$
G_{1}=G_{2}=-G .
$$

From (3.14) and (3.16), it is easy to see that $\bar{f}=\underline{f}=-f$ and $\bar{w}=\underline{w}$. We deduce from (3.13) that

$$
\bar{w}=\frac{b \bar{f}-a}{\bar{f}-1}=\frac{b f+a}{f+1}=\frac{\frac{b(w-a)}{w-b}+a}{\frac{w-a}{w-b}+1}=\frac{w-a b}{w-1},
$$

i.e., $\bar{w}=\frac{w-\lambda}{w-1}$.

Case 2. Suppose that

$$
a-\lambda=-a(a-1), \quad b-\lambda=-b(b-1) .
$$

Then $a^{2}=\lambda$ and $b^{2}=\lambda$. Without loss of generality, we assume

$$
a=\sqrt{\lambda}, \quad b=-\sqrt{\lambda} .
$$

From $B(z)=0, D(z)=0$ and (3.19), we have

$$
\begin{aligned}
& 2 b(b-1)(1-a) G_{1} G_{2}=b(b-1)^{2} G\left(G_{1}+G_{2}\right), \\
& 2 a(b-1)(1-a) G=-a(a-1)^{2}\left(G_{1}+G_{2}\right) .
\end{aligned}
$$


If $a=1$ or $b=1$, we get from (3.19) that $\lambda=1$, which is a contradiction. Then $a \neq 1$ and $b \neq 1$. Noting that $a b \neq 0$, the last two equations mean

$$
\begin{aligned}
& 2(a-1) G_{1} G_{2}=(b-1) G\left(G_{1}+G_{2}\right), \\
& 2(b-1) G=(a-1)\left(G_{1}+G_{2}\right) .
\end{aligned}
$$

We deduce from the above two equations that $\left(G_{1}-G_{2}\right)^{2}=0$, which is $G_{1}=G_{2}$, and both are equal to $\frac{b-1}{a-1} G$ by the last equation. From (3.14) and (3.16), we obtain that

$$
\bar{f}=\frac{b-1}{a-1} f \quad \text { and } \quad f=\frac{b-1}{a-1} f,
$$

which yield $(a-1)^{2}=(b-1)^{2}$, a contradiction since $a+b=0$.

Case 3. Suppose that

$$
a-\lambda=a(a-1), \quad b-\lambda=-b(b-1) .
$$

Then $b^{2}=\lambda$. Since $\lambda \neq 1$, it is easy to see that $a \neq 1$ and $b \neq 1$ by (3.20). Noting that $a b \neq 0$, we get from $B(z)=0, C(z)=0$ and $D(z)=0$ that

$$
\begin{aligned}
& 2(1-a)(a+b) G_{1} G_{2}=(b-1)(a-b) G\left(G_{1}+G_{2}\right), \\
& \left(a^{2}-b^{2}\right)\left[(b-1)^{2} G^{2}-(a-1)^{2} G_{1} G_{2}\right]=4 a b(b-1)(1-a) G\left(G_{1}+G_{2}\right), \\
& 2(b-1)(a+b) G=(a-1)(a-b)\left(G_{1}+G_{2}\right) .
\end{aligned}
$$

Combining (3.21) with (3.22) and (3.23) respectively, we obtain

$$
\begin{aligned}
& (a-b)^{2}(b-1)^{2} G^{2}=(a-1)^{2}\left[(a-b)^{2}+8 a b\right] G_{1} G_{2}, \\
& (b-1)^{2} G^{2}=-(a-1)^{2} G_{1} G_{2} .
\end{aligned}
$$

Then

$$
(a-b)^{2}=-(a-b)^{2}-8 a b,
$$

which is $a+b=0$. Thus $a=-b= \pm \sqrt{\lambda}$. Substituting $a$ by $\pm \sqrt{\lambda}$ in the first equation of (3.20), we get that $\lambda=0$ or $\lambda=1$, both are impossible.

Proof of Theorem 1.2 Let $P(z, w)=\bar{w} \underline{w} w(w-1)-\mu$. Then $P(z, 0)=-\mu \neq 0$. We deduce from Lemma 2.2 that $m(r, 1 / w)=S(r, w)$, and then $N(r, 1 / w)=T(r, w)+S(r, w)$. Thus $\lambda(w)=\rho(w)$. Assume to the contrary that $w$ has two Borel exceptional values $a$ and $b(\neq a)$. Obviously, $a b \neq 0$ by $\lambda(w)=\rho(w)$. Let $f$ be given by (3.13). Then we still have (3.14)-(3.16). Substituting $w=\frac{b f-a}{f-1}$ in (1.3), we obtain

$$
A_{1}(z) e^{4 d z^{n}}+B_{1}(z) e^{3 d z^{n}}+C_{1}(z) e^{2 d z^{n}}+D_{1}(z) e^{d z^{n}}+E_{1}=0,
$$

where

$$
A_{1}(z)=\left[b^{3}(b-1)-\mu\right] g^{2} \bar{g} g_{1} g g_{2},
$$




$$
\begin{aligned}
& \begin{aligned}
B_{1}(z)= & {\left[b^{2}(a+b-2 a b)+2 \mu\right] g \bar{g} g_{1} g g_{2}+\left[a b^{2}(1-b)+\mu\right] g^{2}\left(\bar{g} g_{1}+g g_{2}\right), } \\
C_{1}(z)= & {\left[a b^{2}(a-1)-\mu\right] \bar{g} g_{1} g g_{2}-[a b(a+b-2 a b)+2 \mu] g\left(\bar{g} g_{1}+g g_{2}\right) } \\
& +\left[a^{2} b(b-1)-\mu\right] g^{2},
\end{aligned} \\
& D_{1}(z)=\left[\mu-a^{2} b(a-1)\right]\left(\bar{g} g_{1}+g g_{2}\right)+\left[a^{2}(a+b-2 a b)+2 \mu\right] g, \\
& E_{1}=a^{3}(a-1)-\mu .
\end{aligned}
$$

Lemma 2.3 tells us that all the coefficients of (3.24) vanish. By a similar way to the above, we deduce from $A_{1}(z)=0$ and $E_{1}=0$ that

$$
a^{3}(a-1)=\mu, \quad b^{3}(b-1)=\mu .
$$

Denote $G=g, G_{1}=\bar{g} g_{1}$ and $G_{2}=g g_{2}$. From $B_{1}(z)=0, C_{1}(z)=0$ and (3.25), and noting that $a \neq b$, we have

$$
\begin{aligned}
& (1-2 b) G_{1} G_{2}=(b-1) G\left(G_{1}+G_{2}\right), \\
& (1-2 a) G=(a-1)\left(G_{1}+G_{2}\right) .
\end{aligned}
$$

Since the last two equations are both homogeneous, there exist two non-zero constants $\alpha$ and $\beta$ such that $G_{1}=\alpha G$ and $G_{2}=\beta G$. Then

$$
\begin{aligned}
& (1-2 b) \alpha \beta=(b-1)(\alpha+\beta), \\
& (1-2 a)=(a-1)(\alpha+\beta) .
\end{aligned}
$$

If $\alpha+\beta=0$, then $a=b=\frac{1}{2}$ by (3.26) and (3.27), which is a contradiction. Thus $\alpha+\beta \neq 0$. On the other hand, combining (3.14) with (3.16), we have

$$
\bar{f}=\alpha f, \quad \underline{f}=\beta f,
$$

which yield $\alpha \beta=1$. It follows from (3.26) and (3.27) that

$$
\frac{1-2 b}{1-2 a}=\frac{b-1}{a-1}
$$

which means $a=b$, a contradiction.

\section{Competing interests}

The author declares that he has no competing interests.

\section{Author's contributions}

The author drafted the manuscript, read and approved the final manuscript.

\section{Acknowledgements}

The author would like to thank the referee for his or her valuable suggestions to the present paper. This research was supported by the NNSF of China Nos. 11201014, 11171013, 11126036 and the YWF-14-SXXY-008, YWF-ZY-302854 of Beihang University. This research was also supported by the youth talent program of Beijing No. 29201443. 


\section{References}

1. Hayman, WK: Meromorphic Functions. Clarendon Press, Oxford (1964)

2. Laine, I: Nevanlinna Theory and Complex Differential Equations. de Gruyter, Berlin (1993)

3. Yang, CC, Yi, HX: Uniqueness Theory of Meromorphic Functions. Kluwer Academic, Dordrecht (2003)

4. Chiang, YM, Feng, SJ: On the Nevanlinna characteristic of $f(z+\eta)$ and difference equations in the complex plane. Ramanujan J. 16, 105-129 (2008)

5. Halburd, RG, Korhonen, RJ: Difference analogue of the lemma on the logarithmic derivative with applications to difference equations. J. Math. Anal. Appl. 314, 477-487 (2006)

6. Laine, I, Yang, CC: Clunie theorems for difference and q-difference polynomials. J. Lond. Math. Soc. 76, 556-566 (2007)

7. Halburd, RG, Korhonen, RJ: Finite order solutions and the discrete Painlevé equations. Proc. Lond. Math. Soc. 94, 443-474 (2007)

8. Ronkainen, O: Meromorphic solutions of difference Painlevé equations. Ann. Acad. Sci. Fenn., Math. Diss. 155, 59pp (2010)

9. Chen, ZX, Shon, KH: Value distribution of meromorphic solutions of certain difference Painlevé equations. J. Math. Anal. Appl. 364, 556-566 (2010)

10. Zhang, JL, Yang, LZ: Meromorphic solutions of Painlevé III difference equations. Acta Math. Sin. 57, 181-188 (2014)

11. Zhang, JL, Yi, HX: Properties of meromorphic solutions of Painlevé III difference equations. Adv. Differ. Equ. 2013,256 (2013)

10.1186/1029-242X-2014-330

Cite this article as: Zhang: Meromorphic solutions of Painlevé III difference equations with Borel exceptional values. Journal of Inequalities and Applications 2014, 2014:330

\section{Submit your manuscript to a SpringerOpen ${ }^{\circ}$ journal and benefit from:}

- Convenient online submission

- Rigorous peer review

- Immediate publication on acceptance

- Open access: articles freely available online

- High visibility within the field

- Retaining the copyright to your article 